\title{
Effectiveness of a 4-week rehabilitation program on endothelial function, blood vessel elasticity in patients with chronic obstructive pulmonary disease
}

\author{
Botond Szucs $^{1,2}$, Mate Petrekanits $^{1,2}$, Janos Varga $^{3}$ \\ ${ }^{1}$ PharmaFlight Research and Training Center, Debrecen, Hungary; ${ }^{2}$ Institute of Exercise Physiology and Sport Medicine, University of Physical \\ Education, Budapest, Hungary; ${ }^{3}$ Department of Pulmonary Rehabilitation, National Koranyi Institute for Pulmonology, Budapest, Hungary \\ Contributions: (I) Conception and design: M Petrekanits, J Varga; (II) Administrative support: J Varga; (III) Provision of study materials or patients: M \\ Petrekanits, J Varga; (IV) Collection and assembly of data: B Szucs; (V) Data analysis and interpretation: B Szucs, J Varga; (VI) Manuscript writing: \\ All authors; (VII) Final approval of manuscript: All authors. \\ Correspondence to: Janos Varga, MD, PhD. No. 1, Piheno Street, H-1121 Budapest, Hungary. Email: varga@koranyi.hu.
}

Background: Chronic obstructive pulmonary disease (COPD) may have considerable cardiovascular risk. Physical activity has a paramount role in COPD treatment. Our aim was to evaluate the applicability of arteriograph in COPD and measure the effectiveness of pulmonary rehabilitation on endothelial function.

Methods: A total of 40 patients with COPD $\left(\mathrm{FEV}_{1}: 45.43 \pm 20.20 \%\right.$ pred, BMI: $27.99 \pm 6.98 \mathrm{~kg} / \mathrm{m}^{2}$, male: female was 21:19, age: $65.47 \pm 7.39$ years) participated in a 4-week rehabilitation program. We used a patented, invasively validated Arteriograph. Blood pressure, pulse, augmentation index (AIX), pulse wave velocity (PWV), diastolic area index (DAI) were registered with functional measurements in pulmonary rehabilitation.

Results: Pulmonary rehabilitation was effective in 6 minutes walking distance (6MWD: $335.32 \pm 110.43$ vs. $398.32 \pm 126.21 \mathrm{~m}$ ), maximal inspiratory pressure (MIP: $57.72 \pm 22.69 v s .63 .63 \pm 18.01 \mathrm{cmH}_{2} \mathrm{O}$ ), chest wall expansion (CWE: $2.84 \pm 1.26$ vs. $4.00 \pm 1.76 \mathrm{~cm}$ ), breath holding time (BHT: $25.77 \pm 10.63$ to $29.21 \pm 11.60 \mathrm{sec}$ ) and grip strength (GS: $24.87 \pm 11.88$ vs. $27.03 \pm 11.43 \mathrm{~kg})(\mathrm{P}<0.05)$. Improvement in quality of life was monitored by COPD assessment test marker (CAT: $17.00 \pm 8.49$ vs. $11.89 \pm 7.31, \mathrm{P}<0.05$ ). Systolic $(133.38 \pm 22.15$ vs. $126.48 \pm 20.22 \mathrm{mmHg})$ and diastolic blood pressure $(76.95 \pm 14.37$ vs. $75.4 \pm 12.7 \mathrm{mmHg})$ showed a reduction tendency. Pulse also decreased $(76.95 \pm 14.37$ vs. $72.53 \pm 13.65 \mathrm{bpm})$. AIX levels showed slight improvement $(3.54 \pm 35.59 \%$ vs. $2.93 \pm 30.79 \%) ; 23$ patients peripheral circulation progressed. The PWV data showed abnormal elasticity with minimal change $(11.74 \pm 2.13$ vs. $11.4 \pm 2.73 \mathrm{~m} / \mathrm{s})$; although 20 patients showed improvement. DAI detected slightly diminished coronary circulation with moderate improvement $(43.32 \pm 6.81$ vs. $47.1 \pm 7.01 \mathrm{~m} / \mathrm{s})$.

Conclusions: Elevated arterial stiffness, high PWV turned the COPD patients to the high/very high-risk cluster. Rehabilitation resulted significant improvement in MIP, CWE, BHT, 6MWD, CAT with mild, but favorable changes in blood pressure, pulse, AIX, PWV. As a consequence of the four weeks rehabilitation period overall quality of life improved and cardiovascular risk showed a reduction tendency in COPD.

Keywords: Chronic obstructive pulmonary disease (COPD); pulmonary rehabilitation; endothelial function; cardiovascular risk

Submitted Feb 06, 2018. Accepted for publication Oct 15, 2018.

doi: $10.21037 /$ jtd.2018.10.104

View this article at: http://dx.doi.org/10.21037/jtd.2018.10.104 


\section{Introduction}

In a part of the chronic obstructive pulmonary disease (COPD) patients may have an association with multisystemic inflammation $(1,2)$. It can be manifested in extrapulmonary comorbidities, like respiratory muscle weakness, cardiovascular and cardiac autonomic regulation disorders (3).

Cardiovascular disease is an important factor for death in COPD (4,5). A complex management of patients with COPD involves exercise training as an important part of the rehabilitation (6). Exercise based pulmonary rehabilitation has favorable effects on circulation, metabolism, muscles and overall quality of life (7-10).

Measuring arterial stiffness with pulse wave velocity (PWV) (11) and augmentation index (AIX) are important for cardiovascular risk estimation $(12,13)$. PWV is an independent predictor of mortality (14), and has been widely used in epidemiological (15) and interventional studies (16). PWA provides useful information regarding the mechanical properties of the arterial tree and the ventricular-vascular interaction (17) and can also be used to assess endothelial function (18). A higher value of PWV can be detected in COPD patients $(19,20)$.

Our aim was to evaluate the effectiveness of a 4-week rehabilitation program, focusing on arterial stiffness in patients with COPD. To further assess the condition of the entire cardiac system we used additional parameters: systolic (Sys) and diastolic (Dia) blood pressure, heart rate (HR), diastolic area index (DAI) (21).

\section{Methods}

\section{Study subjects}

A total of 40 patients with COPD $\left[\mathrm{FEV}_{1}: 45.43 \pm 20.20 \%\right.$ pred, age: $65.47 \pm 7.39$ years (range, $51-78$ years), BMI: $27.99 \pm 6.98 \mathrm{~kg} / \mathrm{m}^{2}$, male: female was $\left.21: 19\right]$ participated in this study. All patients gave consent for participation in the study. The pharmacotherapy of patients was based on the international GOLD guideline, mostly the patients were on dual bronchodilator therapy, 5 of them was on $\mathrm{ICS}+\mathrm{LABA}+\mathrm{LAMA}$ combination. The treatment of the co-morbidities, like atherosclerosis, hypertension and diabetes was based on international guideline, also. The study protocol was approved by the Ethical Committee of the National Koranyi Institute for Pulmonology with $25 / 2017$ registration number and the study was registered at ISRCTN registry with ISRCTN13019180 ID. It was
Table 1 Patients' characteristics

\begin{tabular}{|c|c|}
\hline Characteristics $(n=40)$ & Values \\
\hline Age (years) & $65.47 \pm 7.39$ \\
\hline Male: female & $21: 19$ \\
\hline $\mathrm{BMI}\left(\mathrm{kg} / \mathrm{m}^{2}\right)$ & $27.99 \pm 6.98$ \\
\hline $\mathrm{FEV}_{1}$ (\%pred) & $45.43 \pm 20.20$ \\
\hline Hypertension, $\mathrm{n}$ & 35 \\
\hline Diabetes, $\mathrm{n}$ & 12 \\
\hline Atherosclerosis, $\mathrm{n}$ & 30 \\
\hline Cor pulmonale, $\mathrm{n}$ & 10 \\
\hline $\mathrm{pH}$ & $7.43 \pm 0.06$ \\
\hline $\mathrm{pO}_{2}(\mathrm{mmHg})$ & $63 \pm 8$ \\
\hline $\mathrm{pCO}_{2}(\mathrm{mmHg})$ & $39 \pm 3$ \\
\hline
\end{tabular}

$\mathrm{BMI}$, body mass index; $\mathrm{FEV}_{1}$, forced expiratory volume in the first second, $\mathrm{pO}_{2}$, partial pressure of the oxygen, $\mathrm{pCO}_{2}$, partial pressure of the carbon-dioxide.

an observational study using the general management in the Department of Pulmonary rehabilitation as a noninterventional study. Patients' characteristics including blood gas analysis are presented in Table 1. Inclusion criterias were the following: age 40 or above, $\mathrm{FEV}_{1}<70 \%$ pred and $\mathrm{FEV}_{1} / \mathrm{FVC}<0.7$, more than 10 years smoking anamnesis with COPD diagnosis, symptomatic patients in terms of dyspnea and reduction in exercise tolerance, absence of type II respiratory failure, good compliance of the patient. The exclusion criterias were the following: any condition which did not allowed to participate in a training program because of joint disease, coronary disease, heart failure, not a good compliance in the previous treatment of COPD or absence of the motivation to the rehabilitation program.

\section{Measurements}

\section{Pulmonary function}

Lung function test was performed as a post-bronchodilator testing ( $\mathrm{V}_{\max } 229$ and Autobox 6200, Sensormedics) including spirometry measurements based on ATS/ERS guidelines (22). Twenty minutes before testing patients inhaled $400 \mu g$ salbutamol.

\section{Six minutes walking distance (6MWD)}

The $6 \mathrm{MWD}$ was measured at the corridor of our department. Before, during and after walking an oxygen 
saturation and heart rate were measured, and a modified Borg-scale was evaluated. The speed of walking was as fast as possible (23).

\section{Chest wall expansion (CWE)}

CWE means the difference of chest circumferences between deep inspiration and expiration. It is measured at the level of processus xyphoideus (24).

\section{Maximal inspiratory pressure (MIP)}

To evaluate MIP, we used a special digital instrument, Power Breathe K1 (POWERbreathe International Limited). The calculation of diaphragmatic force was based on the patient's height, weight, age and sex (very poor, poor, average, fair, good, very good). Patients were asked to inhale suddenly with maximal force after a maximal exhalation (25).

\section{Breath holding time (BHT)}

BHT displays the severity of COPD. After a maximal inhalation the subjects were asked to hold the breath as long as possible with closed nose and mouth without inhalation (26).

\section{Grip strength (GS) measurement}

Kern hand grip dynamometer (2016 Kern \& Sohn GmbH, Germany) was used to identify the peripheral muscles force (27).

\section{Rebabilitation program}

Patients performed a complex pulmonary rehabilitation programme with chest wall-stretching, controlled breathing techniques and training, as well as personalized exercise training 2-3 times for 20-30 minutes per day by cycling and treadmill for 4 weeks (28-31). 6MWD (23), lung function (22), CWE (24), GS (27), MIP (25) and BHT (26) were measured. The quality of life and dyspnoea were evaluated by COPD assessment test (CAT) marker (32) and mMRC (33).

The breathing exercises were performed on an openair corridor with the special microclimate of the Hospital $(30,31)$. The patients learned controlled breathing and stretching technics, chest and spine mobilization exercises $(30,31)$. The personalized training was performed on exercise bicycles and treadmills with continuous or interval training $(28,29)$. The training set was according to the patients' requirements. The protocol was defined considering the stage of COPD, the actual status of the heart, comorbidities and blood gas values $(30,31)$. The functional measurement was performed at the beginning and at the end of the programme.

Our patients performed respiratory training in the morning, chest wall mobilization, learning controlled breathing techniques (it lasted 30 minutes) in the morning, inhalation, expectoration, smoking cessation and a personalized training. The personalized training was based on continuous or interval type of cycle- and/or treadmill training for 10-30 minutes, 2-3 times a day at a level of $60-80 \%$ of peak work rate (29-31). The duration was 3 weeks. The intensity was between $60-80 \%$ of peak work rate and it was progressive keeping Borg dyspnea scale breathlessness and leg fatigue on grade No. 7 .

\section{Quality of life marker and dyspnea score}

Quality of life by CAT marker (32) and dyspnea by Modified Medical Research Council Dyspnoea Scale (mMRC) were evaluated (33).

\section{Resting measurements' condition}

We used a standardized protocol, all methods were noninvasive and performed at the same time between 9 and 11 in the morning. We performed our resting evaluations on the first and last day of the rehabilitation. Patients were asked not to do any physical activity on days of measurements, the assessing room was separated, quiet and temperature controlled (maintained around $24^{\circ} \mathrm{C}$ ). The patients had rested quietly in supine position for 15 minutes before the Arteriograph measurements.

\section{Measurement of arterial stiffness}

We used Arteriograph (Tensiomed Ltd., Hungary) for overall cardiovascular status, endothelial function, central blood pressure and large arterial characteristics of the patients (34). The Arteriograph is a patented, invasively validated $(21,35)$ oscillometric tool $(35)$, which can collect information about the pulse pressure curve from the upper arm using an upper arm cuff (36). We used a special stopflow method, (stop the perfusion of the brachial artery) eliminates the disturbance of the pressure curve. This method is a non-invasive, we stop the perfusion only for a few seconds. After a ventricular ejection a direct a late systolic wave can be differentiated, this helps to divide the curve into a systolic and diastolic phase. The reflected wave 
Table 2 Functional and quality of life marker parameters

\begin{tabular}{lcc}
\hline Parameter & Before treatment & After treatment \\
\hline FEV $_{1}(\%$ pred $)$ & $45.43 \pm 20.2$ & $45.06 \pm 18.2$ \\
FVC & $75.81 \pm 22.71$ & $74.78 \pm 17.37$ \\
mMRC & $1.86 \pm 0.71$ & $1.63 \pm 0.6^{*}$ \\
MIP $\left(\mathrm{cmH}_{2} \mathrm{O}\right)$ & $57.72 \pm 22.69$ & $63.63 \pm 18.01^{*}$ \\
CWE $(\mathrm{cm})$ & $2.84 \pm 1.26$ & $4.00 \pm 1.76^{*}$ \\
BHT $(\mathrm{sec})$ & $25.77 \pm 10.63$ & $29.21 \pm 11.60^{*}$ \\
GS $(\mathrm{kg})$ & $24.87 \pm 11.88$ & $27.03 \pm 11.43^{*}$ \\
6MWD $(\mathrm{m})$ & $335.32 \pm 110.43$ & $398.32 \pm 126.21^{*}$ \\
CAT & $17.00 \pm 8.49$ & $11.89 \pm 7.31^{*}$ \\
\hline
\end{tabular}

*, $\mathrm{P}<0.05 . \mathrm{FEV}_{1}$, forced expiratory volume in the first second; FVC, forced vital capacity; mMRC, modified Medical Research Council Dyspnoea Scale; MIP, maximal inspiratory pressure; CWE, chest wall expansion; BHT, breath holding time; GS, grip strength; 6MWD, 6 minutes walking distance; CAT, COPD assessment test.

gives information from the periphery, as a composition of all reflective points, it is mainly related to the aortic bifurcation. It is able to collect information about the position and the amplitude of the reflective wave and determine simultaneously the PWV, AIX and DAI, as well as the traditional blood pressure data (systolic and diastolic blood pressure, heart rate).

AIX traces the grade of pulse wave reflection, it basically depicts the state of peripheral circulation. AIX and the peripheral circulation are related with cardiovascular mortality (37). Apart from the arteries elasticity it depends on the vascular resistance of the small blood vessels, arterioles the total peripheral resistance (TPR). The arterial pulse wave has two systolic apex, the first is the ejection caused early systolic wave (P1), the second is the reflected late systolic wave (P2), the difference of $\mathrm{P} 2$ and $\mathrm{P} 1$ compared to the pulse pressure (PP) ratio, represented in percentile is the AIX. AIX can be negative if $\mathrm{P} 2$ is smaller than $\mathrm{P} 1$, or positive if the reflected wave is stronger than the ejection caused wave. Physiologically a negative AIX is optimal, a positive AIX means a continuous extra load on the heart (37-39). AIX is divided into four quartiles: (I) optimal: AIX $<-30 \%$; (II) normal: $-30 \% \leq \mathrm{AIX} \leq-10 \%$; (III) elevated: $-10 \% \leq$ AIX $\leq 10 \%$; (IV) pathological $10 \%<$ AIX. AIX has prognostic value over classic risk factors, what is the reason we used it in our study (35).

To calculate PWV apart from knowing the time of the wave's return we need a distance value (path), which we have to manually insert into the program while taking patient data. The calculation of the difference between the jugulum and the symphysis is able to match aortic length in practice. PWV therefore represents the speed of the pressure wave on the aorta generated by the hearts contraction, which is crucially affected by the elasticity of the aorta (40). PWVao is a solid, independent, proven predictor of death associated with cardiovascular risks $(41,42)$. The threshold limits of PWV are the following: optimal: $\mathrm{PWV}<7 \mathrm{~m} / \mathrm{s}$; normal: $7 \mathrm{~m} / \mathrm{s} \leq \mathrm{PWV} \leq 9.7 \mathrm{~m} / \mathrm{s}$; elevated: $9.7 \mathrm{~m} / \mathrm{s} \leq \mathrm{PWV} \leq 12 \mathrm{~m} / \mathrm{s}$; pathological: $12 \mathrm{~m} / \mathrm{s}<$ PWV.

DAI represents the diastolic proportion of the cardiac cycle. With the use of DAI and DRA (diastolic reflection area) we can get more information about the pressure properties of the left coronary artery, and the work of the atria, the isovolumetric relaxation, the rapid inflow and the diastasis. In healthy individuals the DAI is between $50 \%$ and $60 \%(40)$.

\section{Statistical analysis}

We used paired $t$-test and non-parametric sign test and Wilcoxon test for comparison of patients' characteristics, lung function and exercise physiologic and arterial stiffness variables. The level of the significance was determined as $\mathrm{P}<0.05$ level. Observed data were expressed as $\pm \mathrm{SD}$ in tables. We used the Kolmogorov-Smirnov test for testing the distributions.

\section{Results}

Functional and quality of life parameters at start of the rehabilitation are presented in Table 2. Pulmonary function did not show improvement after the rehabilitation period (Table 2). Significant changes were detected in CWE, BHT, GS, 6MWD, CAT, mMRC (Table 2). MIP results showed significant increment, but the values are still under the physiologic values (Table 2).

Systolic and diastolic blood pressure and resting average pulse showed marginal improvement. Systolic and diastolic blood pressure decreased, as did average resting pulse (Table 3).

The AIX, PWV and DAI has not shown clear, significant improvement. Considering changes on an individual level these parameters show an improvement tendency (Table 3).

The 4-week treatment resulted in not significant, moderate improvement in AIX. AIX data in 6 patients 
improved and turned to a lower cardiovascular risk group (Figure 1). We would like to underline that four of these patients were at the highest risk group at the start of the rehabilitation and turned to the normal level group (Table 3); 17 additional patients AIX improved, although these changes did not achieve the clinical significance. These results show that four weeks of rehabilitation had a favorable impact on peripheral vascular condition. AIX data showed a moderate elevation in peripheral resistance in 17 patients, although AIX detected extreme elevation in peripheral resistance $(+70 \%$ in AIX) in two patients (Table 3, Figure 1).

The PWV data showed abnormal elasticity before

Table 3 Characteristics measured by arteriograph

\begin{tabular}{lcc}
\hline Parameter & Before treatment & After treatment \\
\hline Sys $(\mathrm{mmHg})$ & $133.38 \pm 22.15$ & $126.48 \pm 20.22$ \\
Dias $(\mathrm{mmHg})$ & $76.95 \pm 14.37$ & $75.4 \pm 12.7$ \\
Pulse $(\mathrm{bpm})$ & $76.95 \pm 14.37$ & $72.53 \pm 13.65$ \\
AIX $(\%)$ & $3.54 \pm 35.59$ & $2.93 \pm 30.79$ \\
PWV $(\mathrm{m} / \mathrm{s})$ & $11.74 \pm 2.13$ & $11.4 \pm 2.73$ \\
DAI $(\%)$ & $43.32 \pm 6.81$ & $47.1 \pm 7.01$ \\
\hline
\end{tabular}

Sys, systolic blood pressure; Dias, diastolic blood pressure; AIX, augmentation index; PWV, pulse wave velocity; DAI, diastolic area index. rehabilitation that became congenial after it. In 9 cases the patients PWV data showed significant improvement, they turned to a different category (Table 3). In 11 other cases we measured improvement of PWV, although these did not achieve clinically significant difference. It's important to add, that in 20 patients PWV results showed elevated PWV from the initial results (Table 3).

Slightly diminished coronary circulation was confirmed by DAI results, with minimal and not significant progress after 4 weeks of rehabilitation. DAI results elevated to the normal reference threshold levels in 12 patients, and 10 patients experienced mildly better coronary flow (Table 3 ).

\section{Discussion}

We conducted a clinical research in terms of determining the vascular function in COPD patients and the effectiveness of pulmonary rehabilitation on endothelial function. 6MWD, CWE, MIP, BHT, GS measurement and quality of life marker like CAT and $\mathrm{mMRC}$ were improved by pulmonary rehabilitation. The 4-week rehabilitation program resulted improvement in AIX in more than half of the patients. The PWV data showed abnormal elasticity and it did not change after the rehabilitation program, although $50 \%$ of the patients had better results after the rehabilitation period. DAI detected slightly diminished coronary circulation, which improved moderately in more than half of patients after rehabilitation. Considering the

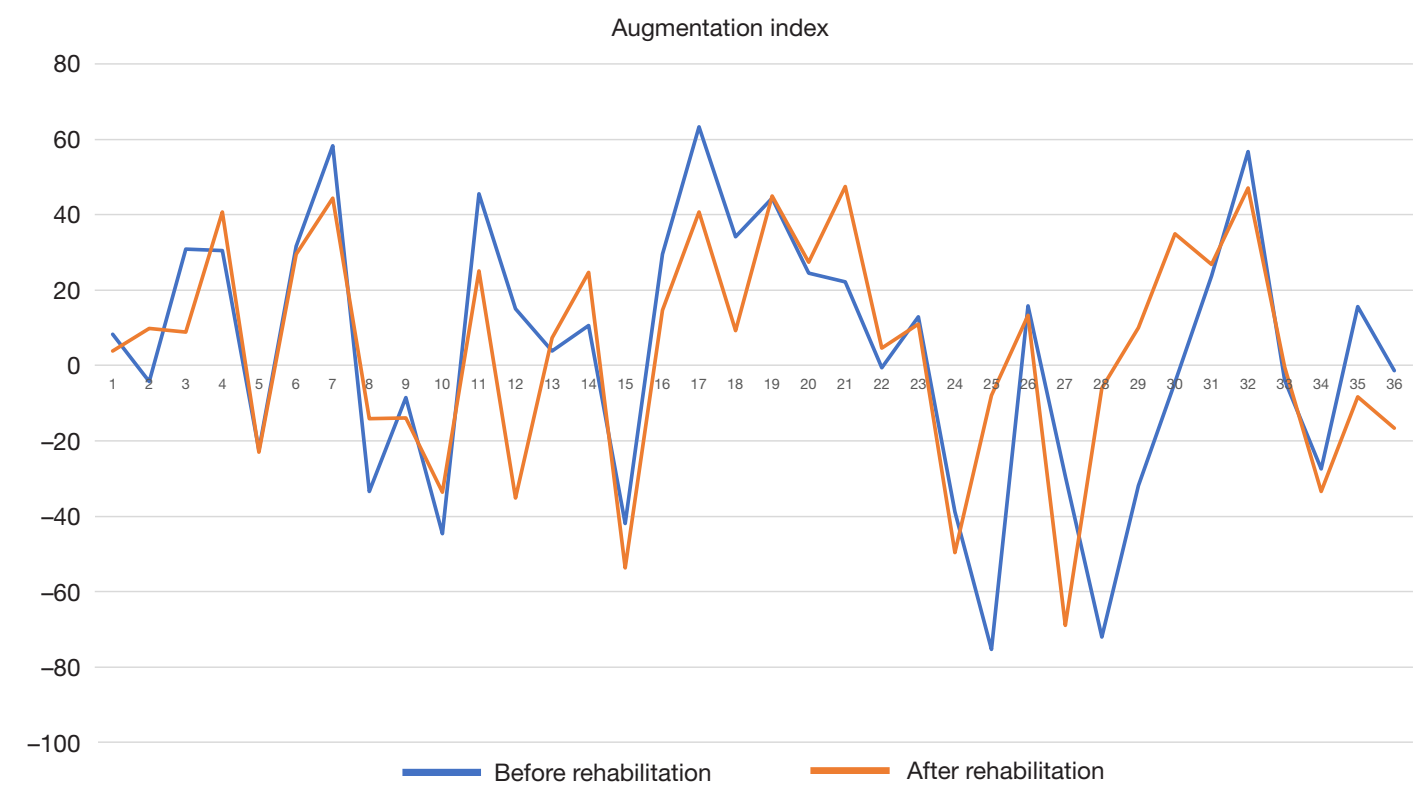

Figure 1 AIX - arterial stiffens characteristic of the vessel before and after rehabilitation on individual basis. AIX, augmentation index. 
short rehabilitation period these improvements show a promising trend.

Arterial stiffness measured by aortic PWV, is an important predictor of cardiovascular mortality so it may improve risk stratification (43). Aortic stiffness can be worsened in COPD patients (43), even without diabetes mellitus or cardiovascular disease comorbidities. Increases in arterial stiffness have systemic non-atherosclerotic consequences including renal, cardiac vasculatures $(44,45)$. We detected abnormal arterial stiffness in general in our COPD patients as well $85 \%$ of our patients were in the elevated/pathological threshold at the start of the rehabilitation program.

Structural and functional components have effect of aortic PWV like the age-dependent distending pressure: mean arterial blood pressure (MAP). There is evidence about the role of chronic inflammation for structural and functional condition of the vessels, anti-inflammatory trials may support the improvement in arterial stiffness (10). There are studies regarding the connection of aortic stiffness and systemic inflammatory mediators in COPD (10) and one study detected increased vascular wall inflammation following the process by positron emission tomography/ computed tomography fluorodeoxyglucose imaging (46).

There is contradictory data about the effect of exercise and/or pulmonary rehabilitation on reductions in aortic PWV in COPD $(47,48)$. In a clinical study there were significant reductions in MAP in relation with moderate improvement in aortic stiffness. Controversy, the recent study by Vanfleteren et al. (49) failed to detect any decrease in MAP in aerobic training program. We need to underline that the patients might not able to maintain a certain intensity level of exercise and medications and timing of the assessment might differ (47).

In a clinical study Bolton and colleagues also suggested, that the absent of improvement in aortic PWV might be related that the patients could not maintain a high exercise intensity level and there was difference in medication, timing of the measurement or they did not able to measure the aortic PWV (48). Significantly higher body mass index, and levels of triglycerides and glucose had effect to the unsuccessful measurement of the aortic PWV (48). Our rehabilitation program was successful based on the functional markers and PWV showed an improvement tendency.

Vanfleteren and coworkers detected high variability in the change in aortic PWV after a pulmonary rehabilitation program (49). We need more clinical observation to understand the underlying mechanisms, including pharmacological and non-pharmacological treatment against of higher level of aortic PWV in COPD patients (49). We found variability in PWV after rehabilitation as well.

Different therapeutic strategies have been investigated to decrease aortic PWV, testing many conditions, including pharmacological, nutritional and lifestyle modification. Contradictory many trials could not find interventions, including exercise to achieve significant reductions in MAP $(48,49)$. Our rehabilitation program needs to be focused in these new elements, also.

In a case-controlled study seventeen stable COPD patients with moderate obstruction and untrained subjects were participated (9). A complex assessment of the body was performed by carotid-radial PWV, pulmonary function, BP, plasmatic biomarkers, walking distance, and peripheral muscle function at start and after 4 weeks in both groups (9). There was no significant different PWV at baseline between the two groups. PWV did not change after 4 weeks in untrained patients, but it reduced with training. There was a correlation between PWV and walking distance, muscle endurance, systolic BP, and fasting glucose in all patients (9). Arterial stiffness may be improved by training-induced reductions in systolic BP and fasting glucose (9). In our study PWV was worse in the COPD group and it showed an improvement tendency after rehabilitation.

Contradictory data are presented about the effectiveness of pulmonary rehabilitation on improvement according to arterial stiffness. Comparison between the effectiveness of supervised outpatient PR program (418 COPD patients) and an unsupervised home-based program (74 COPD patients) were performed in terms of improvement of blood pressure (50). Significant improvements were detected in exercise capacity in supervised group and home-based group, but systolic, diastolic and MAP did not change. Pulmonary rehabilitation in supervised patients with known hypertension and/or cardiovascular disease had no effect on blood pressure (50). We observed an improvement in tendency in blood pressure.

One hundred and fifty-one COPD patients with moderate-to-severe obstruction and 73 healthy agematched control subjects ( 31 smokers and 42 nonsmokers) participated in this study. COPD patients were either exsmokers or current smokers, but the pack-year smoking history was not different to healthy smokers (51). Lower limb perfusion pressure impairment was evaluated by the ankle brachial index and arterial stiffness was assessed by PWV (51). Prevalence of peripheral arterial disease 
was the most frequent in COPD patients than in healthy smokers and nonsmokers (51). Positive correlation between $6 \mathrm{MWD}$ and the degree of peripheral arterial disease and negative correlation between $6 \mathrm{MWD}$ and PWV values were found (51). Tobacco-smoking history and COPD severity had effect on the correlations (51). Peripheral arterial disease needs to be considered on exercise intolerance in COPD (51). We need to focus on arterial dysfunction for the multidisciplinary management in this type of patients (51).

Our study had limitations. The duration of the rehabilitation was shorter than most of the rehabilitation programs, but it was intensive and the patients performed the training 2-3 times per day, so the total amount of rehabilitation work was comparable to previous studies of other colleagues and our group. We may focus on the pulmonary circulation as well to have a complex evaluation of the effect of pulmonary rehabilitation on circulation in these patients.

We conducted a clinical research for the evaluation of vascular impairment in COPD patients. Elevated arterial stiffness, high PWV turned the COPD patients to the high/very high-risk cluster. Pulmonary rehabilitation was effective in terms of exercise tolerance, lung mechanics, chest kinematics, respiratory muscle function and quality of life. Our findings indicate an improvement tendency that rehabilitation had favorable effect on peripheral arterial stiffness, coronary filling and blood pressure response.

\section{Acknowledgements}

None.

\section{Footnote}

Conflicts of Interest: The authors have no conflicts of interest to declare.

Ethical Statement: The study protocol was approved by the Ethical Committee of the National Koranyi Institute for Pulmonology with 25/2017 registration number and written informed consent was obtained from all patients.

\section{References}

1. Spruit MA, Pennings HJ, Janssen PP, et al. Extrapulmonary features in COPD patients entering rehabilitation after stratification for MRC dyspnea grade.
Respir Med 2007;101:2454-63.

2. Barnes PJ, Celli BR. Systemic manifestations and comorbidities of COPD. Eur Respir J 2009;33:1165-85.

3. Decramer M, Rennard S, Troosters T, et al. COPD as a lung disease with systemic consequences clinical impact, mechanisms, and potential for early intervention. COPD 2008;5:235-56.

4. Huiart L, Ernst P, Suissa S. Cardiovascular morbidity and mortality in COPD. Chest 2005;128:2640-6.

5. Curkendall SM, DeLuise C, Jones JK, et al. Cardiovascular disease in patients with chronic obstructive pulmonary disease, Saskatchewan Canada cardiovascular disease in COPD patients. Ann Epidemiol 2006;16:63-70.

6. Yohannes AM, Connolly MJ. Pulmonary rehabilitation programmes in the UK: a national representative survey. Clin Rehabil 2004;18:444-9.

7. Ries AL, Kaplan RM, Myers R, et al. Maintenance after pulmonary rehabilitation in chronic lung disease: a randomized trial. Am J Respir Crit Care Med 2003;167:880-8.

8. Casaburi R, Porszasz J, Burns MR, et al. Physiologic benefits of exercise training in rehabilitation of patients with severe chronic obstructive pulmonary disease. Am J Respir Crit Care Med 1997;155:1541-51.

9. Vivodtzev I, Minet C, Wuyam B, et al. Significant improvement in arterial stiffness after endurance training in patients with COPD. Chest 2010;137:585-92.

10. Gale NS, Duckers JM, Enright S, et al. Does pulmonary rehabilitation address cardiovascular risk factors in patients with COPD? BMC Pulm Med 2011;11:20.

11. Mansia G, De Backer G, Dominiczak A, et al. 2007 ESH-ESC Guidelines for the management of arterial hypertension: the task force for the management of arterial hypertension of the European Society of Hypertension (ESH) and of the European Society of Cardiology (ESC). Blood Press 2007;16:135-232.

12. Laurent S, Cockcroft J, Van Bortel L, et al. European Network for Noninvasive Investigation of Large Arteries: Expert consensus document on arterial stiffness: methodological issues and clinical applications. Eur Heart J 2006;27:2588-605.

13. Jones PW, Agusti AG. Outcomes and markers in the assessment of chronic obstructive pulmonary disease. Eur Respir J 2006;27:822-32.

14. Cavalcante JL, Lima JA, Redheuil A, et al. Aortic stiffness: current understanding and future directions. J Am Coll Cardiol 2011;57:1511-22.

15. Sabit R, Bolton CE, Edwards PH, et al. Arterial stiffness 
and osteoporosis in chronic obstructive pulmonary disease. Am J Respir Crit Care Med 2007;175:1259-65.

16. Vlachopoulos C, Aznaouridis K, O’Rourke MF, et al. Prediction of cardiovascular events and all-cause mortality with central haemodynamics: a systematic review and meta-analysis. Eur Heart J 2010;31:1865-71.

17. Williams B, Lacy PS, Thom SM, et al. Differential impact of blood pressure-lowering drugs on central aortic pressure and clinical outcomes: principal results of the Conduit Artery Function Evaluation (CAFE) study. Circulation 2006;113:1213-25.

18. Vlachopoulos C, O'rourke M. Genesis of the normal and abnormal arterial pulse. Curr Probl Cardiol 2000;25:303-67.

19. Chowienczyk PJ, Kelly RP, MacCallum H, et al. Photoplethysmographic assessment of pulse wave reflection: blunted response to endothelium-dependent beta2-adrenergic vasodilation in type II diabetes mellitus. J Am Coll Cardiol 1999;34:2007-14.

20. Maclay JD, McAllister DA, Mills NL, et al. Vascular dysfunction in chronic obstructive pulmonary disease. Am J Respir Crit Care Med 2009;180:513-20.

21. Baulmann J, Schillings U, Rickert S, et al. A new oscillometric method for assessment of arterial stiffness: comparison with tonometric and piezo-electronic methods. J Hypertens 2008;26:523-8.

22. Quanjer PH, Tammeling GJ, Cotes JE, et al. Lung volumes and forced ventilatory flows. Report Working Party Standardization of Lung Function Tests, European Community for Steel and Coal. Official Statement of the European Respiratory Society. Eur Respir J Suppl 1993;Suppl 16:5-40.

23. Balke B. A Simple Field Test for the Assessment of Physical Fitness. Rep 63-6. Rep Civ Aeromed Res Inst US 1963:1-8.

24. Debouche S, Pitance L, Robert A, et al. Reliability and Reproducibility of Chest Wall Expansion Measurement in Young Healthy Adults. J Manipulative Physiol Ther. 2016;39:443-9.

25. Neumeister $W$, Rasche K, Maas P, et al. Reproducibility of computer-assisted mouth occlusion pressure measurements. Med Klin (Munich) 1996;91 Suppl 2:73-5.

26. Mirsky IA, Lipman E, Grinker RR. Breath holding time in anxiety states. Fed Proc 1946;5:74

27. An KN, Chao NY, Askew LJ. Hand strength measurement instruments. Arch Phys Med Rehabil 1980;61:366-8.

28. Varga J, Porszasz J, Boda K, et al. Supervised high intensity continuous and interval training vs. self-paced training in
COPD. Respir Med 2007;101:2297-304.

29. Varga J, Boda K, Somfay A. Kontrollált és otthoni (nem kontrollált) dinamikus tréning COPD-s betegek rehabilitációjában. Orv Hetil 2005;146:2249-55.

30. Kerti M, Balogh Z, Kelemen K, et al. The relationship between exercise capacity and different functional markers in pulmonary rehabilitation for COPD. Int J Chron Obstruct Pulmon Dis 2018;13:717-24.

31. Vagvolgyi A, Rozgonyi Z, Kerti M, et al. Effectiveness of perioperative pulmonary rehabilitation in thoracic surgery. J Thorac Dis 2017;9:1584-91.

32. Jones PW, Tabberer M, Chen WH. Creating scenarios of the impact of COPD and their relationship to COPD Assessment Test (CAT) scores. BMC Pulm Med 2011;11:42.

33. Launois C, Barbe C, Bertin E, et al. The modified Medical Research Council scale for the assessment of dyspnea in daily living in obesity: a pilot study. BMC Pulm Med 2012;12:61.

34. Shirwany NA, Zou MH. Arterial stiffness: a brief review. Acta Pharmacol Sin. 2010;31:1267-76.

35. Horváth IG, Németh A, Lenkey Z, et al. Invasive validation of a new oscillometric device (Arteriograph) for measuring augmentation index, central blood pressure and aortic pulse wave velocity. J Hypertens 2010;28:2068-75.

36. Boutouyrie P, Revera M, Parati G. Obtaining arterial stiffness indices from simple arm cuff measurements: the holy grail? J Hypertens 2009;27:2159-61.

37. Weber T, Auer J, O'Rourke MF, et al. Arterial Stiffness, Wave Reflections, and the Risk of Coronary Artery Disease. Circulation 2004;109:184-9.

38. Nürnberger J, Keflioglu-Scheiber A, Opazo Saez AM, et al. Augmentation index is associated with cardiovascular risk. J Hypertens 2002;20:2407-14.

39. Westerbacka J, Wilkinson I, Cockcroft J, et al. Diminished Wave Reflection in the Aorta. Hypertension 1999;33:1118-22.

40. Reference Values for Arterial Stiffness' Collaboration. Determinants of pulse wave velocity in healthy people and in the presence of cardiovascular risk factors: 'establishing normal and reference values. Eur Heart J 2010;31:2338-50.

41. Willum-Hansen T, Staessen JA, Torp-Pedersen C, et al. Prognostic Value of Aortic Pulse Wave Velocity as Index of Arterial Stiffness in the General Population. Circulation 2006;113:664-70.

42. Blacher J, Asmar R, Djane S, et al. Aortic Pulse Wave Velocity as a Marker of Cardiovascular Risk in 
Hypertensive Patients. Hypertension. 1999;33:1111-7.

43. Mitchell GF, Hwang SJ, Vasan RS, et al. Arterial stiffness and cardiovascular events: The Framingham Heart Study. Circulation 2010;121:505-11.

44. Garnier AS, Briet M. Arterial Stiffness and Chronic Kidney Disease. Pulse (Basel) 2016;3:229-41.

45. Quinn U, Tomlinson LA, Cockcroft JR. Arterial stiffness. JRSM Cardiovasc Dis 2012;1.

46. Coulson JM, Rudd JH, Duckers JM, et al. Excessive aortic inflammation in chronic obstructive pulmonary disease: an 18F-FDG PET pilot study. J Nucl Med 2010;51:1357-60.

47. Smith MC, Wrobel JP Epidemiology and clinical impact of major comorbidities in patients with COPD. Int J Chron Obstruct Pulmon Dis. 2014;9:871-88.

48. Bolton CE, Gale NS, Cockcroft JR. Exercise and

Cite this article as: Szucs B, Petrekanits M, Varga J. Effectiveness of a 4-week rehabilitation program on endothelial function, blood vessel elasticity in patients with chronic obstructive pulmonary disease. J Thorac Dis 2018;10(12):64826490. doi: $10.21037 /$ jtd.2018.10.104 cardiovascular benefit in subjects with COPD: the need for randomised trials. Eur Respir J 2014;44:263-4.

49. Vanfleteren LE, Spruit MA, Groenen MT, et al. Arterial stiffness in patients with COPD: the role of systemic inflammation and the effects of pulmonary rehabilitation. Eur Respir J 2014;43:1306-15.

50. Canavan JL, Kaliaraju D, Nolan CM, et al. Does pulmonary rehabilitation reduce peripheral blood pressure in patients with chronic obstructive pulmonary disease? Chron Respir Dis 2015;12:256-63.

51. Castagna O, Boussuges A, Nussbaum E, et al. Peripheral arterial disease: an underestimated aetiology of exercise intolerance in chronic obstructive pulmonary disease patients. Eur J Cardiovasc Prev Rehabil 2008;15:270-7. 